\title{
Frequency Analysis of Premature Ventricular Contraction Using 3-D MCG Measurements
}

\author{
Koichiro Kobayashi \\ Applied Superconductivity Research Laboratory, Tokyo Denki University, Muzai-Gakuendai Inzai Chiba, 270-1382, Japan \\ Yoshinori Uchikawa \\ College of Science and Engineering, Tokyo Denki University, Ishizaka Hatoyama Hiki-gun Saitama, 350-0394, Japan
}

\begin{abstract}
We carried out three-dimensional (3-D) vector measurcments of the magnetocardiogram(MCG) on a subject with premature ventricular contraction (PVC) and ten normal subjects using a 39-channel SQUID system. This SQUID magnetometer can detect magnetic field components perpendicular to the chest wall (Bz) and tangential to the chest wall (Bx,By) simultaneously. We used principal component analysis in order to compare the normal sinus rhythm of the PVC patient with the sinus rhythm of ten normal sabjects. The results showed that the frequencies in the MCG waveforms of the PVC subject were dominated by lower frequencies (less than $10 \mathrm{~Hz}$ ) than in normal MCGs, and the frequency distribution of $B x$ was different from those characteristic of $\mathrm{Bz}$ and $\mathrm{By}$.
\end{abstract}

Index Terms - three-dimensional vector measurement, magnetocardiogram, premature ventricular contraction, frequency analysis, principle component analysis

\section{INTRODUCTION}

Premature ventricular contraction (PVC) is one of sudden death factors, it is important to explain this detailed mechanism. Whereas biomagnetic field measurement is widely used the magnetic field perpendicular to the body surface. There are a few reports concerning with a 3-D vector measurement[1]-[5]. We have developed a 39channel SQUID system consisting of thirteen 3-D secondorder gradiometers $(12$ measurement positions $(4 \times 3)$ and a noise measurement) for vector measurement of the MCG. This SQUID magnetometer can detect magnetic field components perpendicular to the chest wall $(\mathrm{Bz})$ and tangential to the chest wall (Bx,By) simultaneously[6],[7]. We carried out three-dimensional vector measurements of magnetocardiogram (MCG) on a subject with PVC and ten

Manuscript received May 26, 1999

K. Kobayashi, e-mail;kobaya@asrl.dendai.ac.jp Tel;+81-476-46-8219, Fax;+81-476-8237 normal subjects. We used principal component analysis in order to compare the normal sinus rhythm of the PVC patient with the sinus rhythm of ten normal subjects.

\section{METHOD}

Fig.1 shows the configuration of a 3-D second-order gradiometer and locations of the measurement positions on a subject's chest. MCG and ECG (lead II) of a PVC and ten normal subjects were measured by this system. There were twenty-four measurement positions ( 2 scanning, fixed circle in Fig.1) on the chest wall and a band-pass filter was used in the range of 0.1 to $80 \mathrm{~Hz}$. All magnetic data were averaged for fifty measurements at each position. The reference signal for averaging was R-wave of ECG. Waveforms obtained from each position were calculated by the fast Fourier transform (FFT) for each magnetic field component, then a covariance (equation 1 ) and its matrix (equation 2) were calculated from these twenty-four frequency characteristics $\left(B_{i}\left(\omega_{m}\right)\right)$, where $M$ is analyzed maximum frequency, $i$ and $j$ are measurement positions, $\bar{B}_{i}$ is mean value of $B_{i}, p$ is total number of measurement positions. This matrix was analyzed by principal component analysis (PCA) with equation 3 , where $\lambda$ is eigenvalue, $I$ is unit matrix. Finally, the score $\left(\mathrm{Z}_{\mathrm{x}}\left(\omega_{\mathrm{ro}}\right)\right)$ defines as equation 4 and score proportion $\left(\mathrm{F}_{k}\left(\omega_{\mathrm{m}}\right)\right)$ defines as equation 5 , where $l_{k}$ is eigenvector, $k$ is $k$-th principal component.

$$
\begin{aligned}
& \sigma_{i j}=\frac{1}{M} \sum_{m=1}^{M}\left(B_{i}\left(\omega_{m}\right)-\bar{B}_{i}\right)\left(B_{j}\left(\omega_{m}\right)-\overline{B_{j}}\right) \\
& \Sigma=\left[\begin{array}{cccc}
\sigma_{11} & \sigma_{12} & \cdots & \sigma_{1 p} \\
\sigma_{21} & \sigma_{22} & \cdots & \sigma_{2 p} \\
\vdots & \vdots & \ddots & \vdots \\
\sigma_{p 1} & \sigma_{p 2} & \cdots & \sigma_{p p}
\end{array}\right] \\
& |\Sigma-\lambda I|=0
\end{aligned}
$$

0018-9464/99\$10.00 @ 1999 IEEE 


$$
\begin{aligned}
& Z_{k}\left(\omega_{m}\right)=\sum_{i=1}^{R}\left[l_{k} \times\left(B_{i}\left(\omega_{m}\right)-\bar{B}_{i}\right)\right] \\
& F_{k}\left(\omega_{m}\right)=\frac{Z_{k}\left(\omega_{m}\right)}{\sum_{m=1}^{M} Z_{k}\left(\omega_{m}\right)} \times 100
\end{aligned}
$$

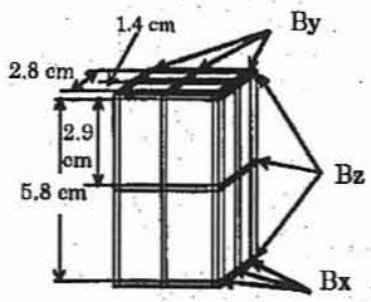

(a) a 3-D second-order gradiometer

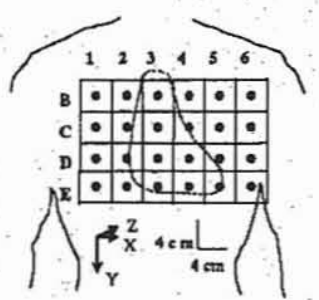

(b) measurement positions econd-order gradiometer Fig.1 Coordinate system and a 3-D second-order gradiom
and measurement positions on a subject's chest.

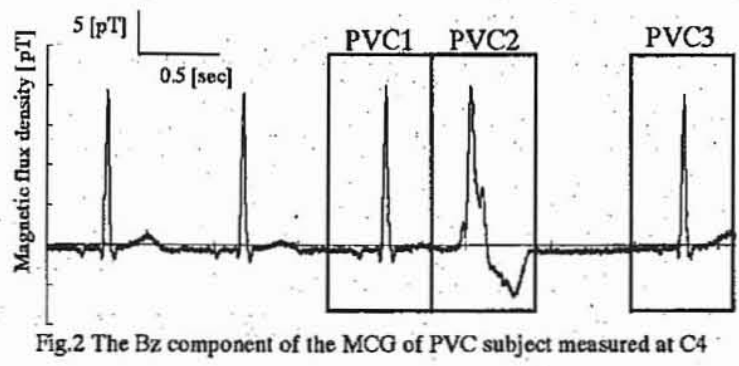

\section{RESULTS AND DISCUSSIONS}

Fig.2 shows an example of the MCG waveform $(\mathrm{Bz})$ recorded from the PVC subject at position C4 in Fig.4(a). To find PVC factor induced by ectopic excitement, the MCG of the PVC subject was classified into four types;
PVC2: Premature Ventricular Contraction, PVC(NSR): normal sinus rhythm, PVC1: heartbeat preceding PVC, PVC3: heartbeat following PVC.

Fig. 3 shows the results of PCA comparing the normal sinus rhythm of the PVC patient (PVC(NSR)) with the sinus rhythm of ten normal subjects. The frequency distribution of score proportion of the PVC(NSR) was dominated by lower frequencies (less than $10 \mathrm{~Hz}$ ) than in normal MCGs, and the frequency distribution of score proportion of $\mathrm{Bx}$ was different from those characteristic of $\mathrm{Bz}$ and By. Table 1 shows the correlation coefficient $(\gamma)$ of the frequency distribution of the score proportion between the perpendicular component and tangential component for each subject. We found that $\gamma$ of the normal sinus rhythm of PVC patient was different from that of normal subjects (BzBx: $\gamma=0.51$ ).

Fig. 4 shows the results of PCA about four types of PVC (NSR,1,2,3) heartbeats. Table 2 shows the correlation coefficient $(\gamma)$ of the frequency distributions of score proportion between PVC(NSR) and PVC1, PVC2, PVC3 for premature ventricular contraction. The frequency distributions $(\mathrm{Bz}, \mathrm{By})$ of score proportion of PVC(NSR) showed a similar pattern as that of $\operatorname{PVC1}(\mathrm{Bz}: \gamma=0.99$, By: $\gamma=0.99)$ and PVC3(Bz: $\gamma=0.98$, By: $\gamma=0.98)$. On the other band, the frequency distribution (Bx) of score proportion of PVC(NSR) was similar to PVC3(Bx: $\gamma=0.99)$, but it differed from that of PVC1(Bx: $\gamma=0.72)$.

Table 1 Correlation coefficiont of the frequency distributions of the
score proportion between the perpendicular component
and tangential component for each subject.
\begin{tabular}{|c|c|c|}
\hline & Normal & PVC(NSR) \\
\hline $\mathrm{Bz}-\mathrm{Bx}$ & 0.93 & 0.51 \\
\hline $\mathrm{Bz}-\mathrm{By}$ & 0.95 & 0.98 \\
\hline
\end{tabular}

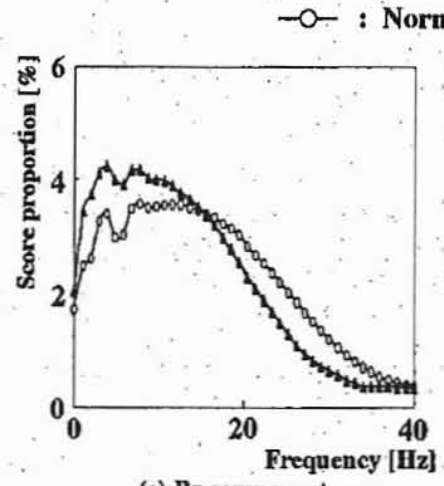

(a) Bz component

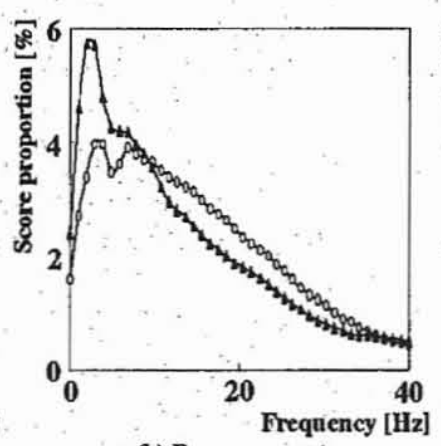

(b) Bx component

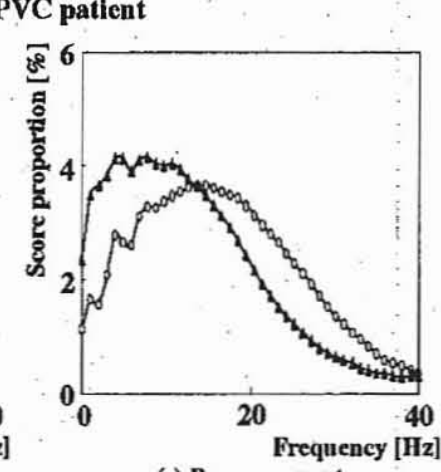

(c) By component

Fig.3 Results of principal component analysis for PVC(NSR) and normal subjects. 


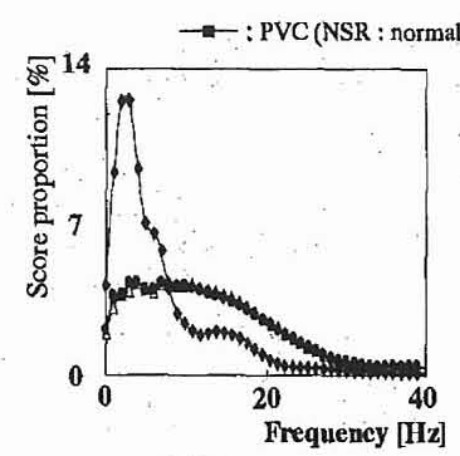

(a) Bz component

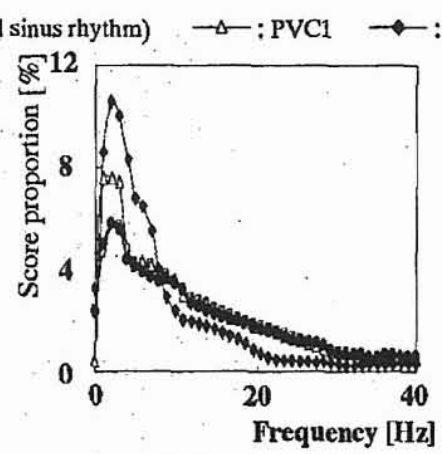

(b) Bx component

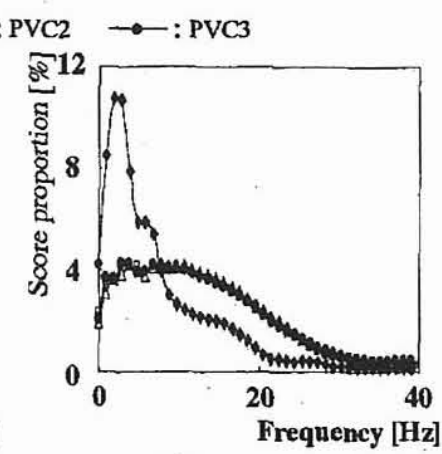

(c) By component

Fig.4 Results of principle component analysis for PVC.

Table 2 Correlation coefficient of the frequency distributions of the score proportion between PVC(NSR) and PVC1,2,3 for premature ventricular contraction.

\begin{tabular}{|l||c|c|c|}
\hline & $\mathrm{Bz}$ & $\mathrm{Bx}$ & $\mathrm{By}$ \\
\hline \hline PVC(NSR)-PVC1 & 0.99 & 0.72 & 0.99 \\
\hline PVC(NSR)-PVC2 & -0.08 & 0.51 & -0.08 \\
\hline PVC(NSR)-PVC3 & 0.98 & 0.99 & 0.98 \\
\hline
\end{tabular}

\section{CONCLUSION}

We carried out three-dimensional vector measurements of magnetocardiogram on a subject with premature ventricular contraction and ten normal subjects. We used principal component analysis in order to compare the normal sinus rhythm of the PVC patient with the sinus rhythm of ten normal subjects. The results showed that the frequency distribution of score proportion of the PVC(NSR) was dominated by lower frequencies (less than $10 \mathrm{~Hz}$ ) than in normal MCGs, and the frequency distribution of score proportion of $\mathrm{Bx}$ was different from those distributions of $\mathrm{Bz}$ and $\mathrm{By}$. The frequency distribution of score proportion of PVC(NSR:normal sinus rhythm) was similar to PVC3(heartbeat following PVC), but it differed from that of PVC1(heartbeat preceding PVC). The study shows promise in detecting components in the normal sinus rhythm in PVC subject, not seen in normal subjects.

\section{ACKNOWLEDGMENTS}

The author thanks M.D. S. Yakubo in School of Medicine, Nihon University for discussion and suggestions.
This study has been supported by a grant from the Advance Technology Research and the Center for Research of Tokyo Denki University, the Japan Ministry of Education, Science and Culture Grant-in-Aid for Scientific Research.

\section{REFERENCES}

[1] A. Rosen, G.T. Inouye : " A study of the vector magnetocardiographic waveform", IEEE Trans. Biomed. Engng. BME-22, 167-174 (1975)

[2] J.P. Wikswo, W.M. Fairbank : "Application of superconducting magnetometers to the measurement of the vector magnetocardiogram", IEEE Trans. Magnetios, MAG13, 354-357 (1977)

[8] J.A.V. Malmivuo, J.P. Wikswo: "A new practical lead system for vector magnetocardiography.”, Proc. IEEE, 65, 809-811 (1977)

[4] M. Seppanen, T. Katila, T. Tuomisto, T. Varpula : " Measurement of biomagnetic fields using multichannel superconducting magnetometer techniques", IL NUOVO CLMENTO, Vol.2, 166-174 (1983)

[5] K. Shirae, H. Furukawa, M. Katayama: "Measurements and characteristics of vector magnetocardiography", Biomagnetism 87, Tokyo Denki Univ. Press, Tokyo, 294-297 (1987)

[6] Y. Uchikawa, K. Kobayashi, M. Kotani : "Application to discrimination of multi-sources to somatosensory evoked field using 3-dimensional second-order gradiometer.", Biomagnetism: Fundamental Research and Clinical Applications, Elsevier Science, IOS Press, 389-393 (1995)

[7] K. Kobayashi, Y. Uchikawa: "Estimation of multiple souroes using a three-dimensional vector measurement of a magnetoencephalogram", Journal of Applied Physics, Vol.83, No.11, Part 2, 6462-6464 (1998) 